\title{
CONOCIMIENTO Y APLICABILIDAD DE LA BIOÉTICA: UNMSM - PERÚ Y CULAGOS/GUADALAJARA - MÉXICO
}

\author{
KNOWLEDGE AND APPLICABILITY OF BIOETHICS: \\ SAN MARCOS UNIVERSITY- PERU AND CULAGOS / GUADALAJARA - MEXICO \\ Ana María Gutiérrez HubY* \\ Docente Principal de la Facultad de Ciencias Contables \\ Universidad Nacional Mayor de San Marcos-UNMSM / Lima-Perú \\ Ma Eugenia Amador Murguia** \\ Docente de la Universidad de Guadalajara - CULAGOS / México \\ [Recepción: Marzo de 2015/ Conformidad: Abril 2015]
}

\section{RESUMEN}

Este artículo trata sobre el conocimiento y aplicabilidad de la bioética en los Centros Universitarios de San Marcos y Los Lagos-CULAGOS / Universidad de Guadalajara. Responde a una investigación básica descriptiva aplicativa. El objetivo, pretende desarrollar en los alumnos, razonamiento bioético para su desenvolvimiento y toma de decisiones frente a conflictos éticos que se le presenten. También determina metodologías y estrategias para incorporar esta disciplina en el diseño curricular e incluirla en la formación integral de futuros gestores empresariales. Por otra parte, determina que a nivel institucional en ambos centros, no armoniza lo declarado en sus respectivas misiones con la ejecución del perfil del estudiante. Se evidencia sí, el compromiso institucional que la universidad tiene como entidad pública, lo que implica trabajar desde la perspectiva de los derechos, la defensa de una educación para la vida y la sociedad, el reconocimiento de derechos ciudadanos, la protección de toda forma de vida y del medio ambiente; así como, que involucra a la bioética para formación integral del ser humano generándole conocimientos con sentido social. Concluye con la necesidad de incorporar esta disciplina a la educación empresarial para que oriente establecimiento de principios en la formación de estudiantes de negocios.

\section{Palabras clave:}

Bioética; formación en bioética; educación con bioética.

\begin{abstract}
This article is about the knowledge and applicability of bioethics at the University Centers of San Marcos and Los Lagos- CULAGOS/University of Guadalajara. This is a basic descriptive applicative research. The goal is to develop in students, bioethical reasoning for their improvement and decision making when facing ethical conflicts that may arise. It also determines methods and strategies to incorporate this discipline in the curriculum and include it in the integral formation of future business managers. Furthermore, we state that at the institutional level in both centers, what is stated in their respective missions does not harmonize with the implementation of the student profile. It is evidenced, the institutional commitment that the university has as a public entity, which implies working from the perspective of rights, the defense of education for life and society, recognition of civil rights, protection of all forms of life and the environment; also the involvement of bioethics for the integral formation of the human being generating knowledge with social sense. We conclude with the need to incorporate this discipline to business education for the establishment of principles to guide the formation of business students.
\end{abstract}

\section{Keywords:}

bioethics; training in bioethics; bioethics education.

\footnotetext{
* Doctora en Administración. Magister en Gestión Empresarial - UNMSM. Email: anamaria_01053@yahoo.es.

** Docente de la Universidad de Guadalajara - Centro Universitario de Los Lagos. México.

*** Colaboradora: Elsa Violeta Rafael Díaz.
} 


\section{INTRODUCCIÓN}

Las formas de convivencia en las que actualmente, se desenvuelve nuestra sociedad por influencia de la aceleración de los avances tecnológicos y científicos así como los cambios económicos, políticos y sociales, acontecidos a nivel mundial, han dado lugar a que esta haya y siga experimentando transformaciones en todos los órdenes, siendo el tecnicismo y el sentido materialista e individualista de los habitantes, las principales características que más distinguen a esta sociedad, reparándose en una sociedad que poco a poco va perdiendo el rumbo en su identidad, lo que se refleja principalmente, en una preocupante crisis de valores en sus individuos y grupos sociales, lo cual viene agudizándose, afectando significativamente la convivencia familiar y social.

Si bien, ahora viene consolidándose la importancia de la percepción de ser conscientes en la gestión de los activos intangibles para legitimarse a nivel social y crear valor más allá de los resultados económicosy financieros ahora es cada vez más usual, cuando nos referirnos a la empresa, abordar conceptos de responsabilidad social, proyección social y compromiso con el entorno; esto ha permitido que ahora las organizaciones empresariales reflexionen acerca de su rol y actuación en la sociedad si están generando un desarrollo que asegure el bienestar de todos con los que se relacionan, si están contribuyendo a incrementar el riesgo de vida en el planeta, si su despliegue contribuye a generar nuevas oportunidades de empleo y de negocios que faciliten la reducción de la pobreza, sobre qué están haciendo para mitigar, reducir o eliminar esos riesgos y si acaso es suficiente llevar adelante solo acciones filantrópicas.

En este sentido, la bioética va más allá de solo el mantenimiento de una conducta justa y deseable de las empresas, que es lo que se promueve con la responsabilidad social empresarial, se trata de tomar conciencia sobre esta y no asumirla como el cumplimiento de normas o imposiciones, sino, con un verdadero sentido de compromiso empresarial de contribuir con el desarrollo, bienestar y el mejoramiento de la calidad de vida de los empleados, sus familias y la comunidad en general, teniendo la capacidad de valorar las consecuencias que tienen en la sociedad las acciones y decisiones que son tomadas para lograr los objetivos y metas propias de estas organizaciones.

La educación para la bioética se constituye en una herramienta para sus fines de adoctrinamiento por esta razón, es preciso reflexionar acerca del rol de la educación y en particular, la educación universitaria en el proceso de transformación del país, considerando que en ella, también impactaron los cambios sociales producidos, un reflejo de ello, ha sido la reducción en los currículos de asignaturas que aportan a la formación del hombre, sustituyéndolos por asignaturas de orientación tecnócrata dejando de lado, el sentido amplio del término educar que busca la elevación del hombre a su máxima expresión, poniendo a su alcance los medios más adecuados para lograr su desarrollo.

\section{LA BIOÉTICA Y SU RELACIÓN CON LA DINÁMICA EMPRESARIAL}

Abordar el concepto de bioética en el contexto empresarial implica, necesariamente, referirnos a la Responsabilidad Social Empresarial (RSE).

En este sentido, las operaciones empresariales adquieren aplicabilidad en las dimensiones teóricas de la empresa, lo cual implica agregar valor a sus actividades frente a la comunidad, cumplir con la legislación vigente, siendo responsables por los impactos de sus decisiones sobre la sociedad y el medio ambiente, partiendo de ello, la reflexión en torno a la forma de cómo gestionar los negocios en el marco de los principios bioéticos de: autonomía, no maleficencia, beneficencia y justicia, considerando que en general, el eje primordial de la bioética lo constituyen las relaciones persiguiendo el objetivo de que éstas se lleven a cabo con ética .

Cabe advertir que, en el Perú y a nivel de Latinoamérica, las empresas están logrando el consenso de que el papel de la empresa en la sociedad está en hacer negocios que la beneficien pero también a la sociedad se le está reconociendo que las partes interesadas o afectadas deben ser parte de sus preocupaciones, se viene reconociendo la necesidad de una visión más amplia de largo plazo no obstante la realidad es que el desarrollo de esta práctica todavía dista mucho de esta percepción. 
Vale decir en este sentido, que aún quedan por responder qué debe esperarse del comportamiento de las empresas, qué significa actuar éticamente, si las empresas se relacionan con una diversidad de actores, grupos o partes de la sociedad con los que interactúa no solo a través de la compra y venta de productos y servicios, sino también, mediante dinámicas laborales y la publicidad, si esas relaciones se dan en el marco de normas y leyes que no cubren todos los aspectos, entonces la ética es una zona gris que abre la posibilidad de actuar de acuerdo a decisiones basadas en principios y valores que están más allá de lo establecido por ley.

\section{EL ROL DEL ESTADO FRENTE A LA BIOÉTICA Y LA RESPONSABILIDAD SOCIAL}

El Estado como principal responsable y garante del bienestar, protección y salud general de sus ciudadanos, se constituye en el primer agente de protección de sus derechos, concretando su responsabilidad con el establecimiento de normas que regulan la creación $\mathrm{y}$ funcionamiento de entidades dedicadas a ayudar a resolver las carencias de la sociedad en todos los ámbitos y estratos socio económicos, aplicando los criterios y principios de respeto a la persona humana, justicia y no maleficencia.

En lo que respecta a la empresa, varios de los aspectos contenidos en la concepción de la responsabilidad social empresarial ya están regulados a través de normas o procedimientos que conforman las políticas generales de la Responsabilidad Social Empresarial, como por ejemplo, las leyes laborales o las disposiciones ambientales mediante las cuales se asegura, en el caso de las empresas, que estas desarrollen prácticas de responsabilidad social.

Por medio de esta entidad, el Estado cumple con resolver la necesidad de la persona humana de buscar su desarrollo, la cual lo consigue en este espacio al situarse en el seno de la racionalidad científica donde se cultiva la ciencia, a través de la aplicación científica y la teoría pura con una apertura y comunicación con todas las vertientes del pensamiento.

\section{LA REPONSABILIDAD SOCIAL EN LA UNIVERSIDAD}

La definición que dio origen a la norma internacional ISO 26000: Guía sobre Responsabilidad Social refiere que "... la responsabilidad "social” es responsabilidad de cada organización por los impactos sociales y ambientales que genera", de acuerdo con esta definición, Vallaeys (2013) refiere que obviamente las universidades no pueden alejarse de la reflexión social de la responsabilidad universitaria, no solo, porque ellas también son organizaciones, sino porque además, les toca formar a los futuros profesionales que laborarán en las empresas, a los futuros ciudadanos que tendrán que promover democráticamente los derechos humanos y a los futuros funcionarios que tendrán a su cargo el bien común en el mundo globalizado.

Vallaeys (2014) ha agrupado los impactos que genera la universidad en la sociedad en cuatro aspectos: impactos de funcionamiento organizacional, impactos educativos, impactos cognitivos y epistemológicos e impactos sociales, aduciendo que son precisamente estos impactos, los que generan la gestión de responsabilidad social universitaria, manifestándola a través de los siguientes Ejes:

Eje 01: La gestión socialmente responsable de la propia organización, con respecto a su clima laboral, los recursos humanos, los procesos democráticos internos y el cuidado del medio ambiente.

Eje 02. La gestión socialmente responsable de la formación académica y la pedagogía, tanto en sus temáticas, organización curricular como metodologías didácticas.

Eje 03. La gestión socialmente responsable de la producción y difusión del saber, la investigación y los modelos epistemológicos promovidos desde el aula.

Eje 04. La gestión socialmente responsable de la participación social en el desarrollo humano sostenible de la comunidad.

Estos ejes constituyen la agenda para la gestión de responsabilidad social por parte de la universidad. 
Figura No 01:

Impactos como Ejes de la Gestión de la Responsabilidad Social Universitaria (RSU)

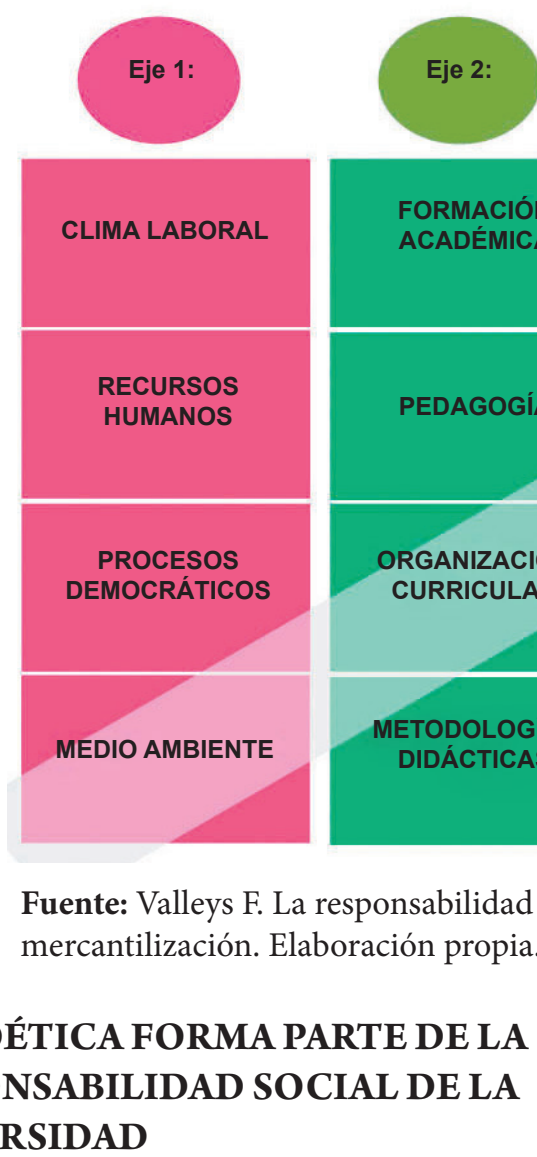

En la era de una sociedad del conocimiento, tal como refiere la UNESCO (2009, citado por Ramos) “... se precisa de la diversidad en los sistemas de educación superior compuestos por una serie de instituciones con variedad de mandatos y dirigidos a diferentes tipos de estudiantes" se reafirma así, su demanda social con una mayor acción por parte de las instituciones universitarias para posibilitar la construcción de conocimientos para su población.

En este sentido, a la universidad le compete reconocer la amplitud del alcance y el impacto positivo o negativo que pueda generar a partir del cumplimiento de su objeto social adquiriendo una gran responsabilidad frente a la sociedad en general y con el Estado a quien representa en su condición de institución de servicio público. Asimismo, tiene la responsabilidad social de contribuir a resolver los problemas y necesidades del país en todos los ámbitos y estratos socioeconómicos, a través de la formación y de la generación del conocimiento para la sociedad.
En este marco, la calidad de la universidad se refleja en la calidad de sus egresados en cuanto a lo profesional, conceptual e institucional así como, en lo ético y lo moral, con principios que le ayudan a comprender su función social desde un punto de vista más humano y menos instrumentaly es, precisamente el valor de defensa a la vida en todas sus manifestaciones, lo que debe acompañar la construcción de conocimientos debiendo ser prioridad dentro de la formación profesional de hoy.

\section{BIOÉTICA: EL POR QUÉ DEBE SER INCORPORADA AL CURRÍCULO UNIVERSITARIO Y SU LEGITIMACIÓN}

Rescatando lo referido por Espejo (2004) la incorporación de la bioética al currículo universitario se legitima desde tres ópticas: del Estado, la bioética misma y la institución.

A partir del Estado, al ser este responsable de velar por una convivencia libre, pacífica y solidaria de sus ciudadanos, le corresponde garantizar los recursos para que estos conceptos se lleven a cabo. 
A partir de la bioética misma familiariza al alumno con la dimensión ética de la vida dada la crisis de los valores existente, haciendo que estos se fortalezcan así como los principios en su relación con el medio empresarial, la sociedad y la naturaleza. Finalmente, en función de la institución educativa, la legitimación de la formación en bioética encuentra su razón en la función misional de la formación, mediante ésta se ayudará al alumno a situarse con cierta madurez ante los sistemas de valores que le presenta el ámbito socio cultural.

Este aporte pedagógico para la enseñanza de la bioética se concreta a su vez, en tres aspectos principales: propicia la comprensión de la tradición cultural aportando a su mayor conocimiento, ayudando a la reafirmación de la propia identidad, se encuentra fundamentada en la dignidad humana, es decir, si un alumno es consciente válidamente de su total dignidad, significará que habrá cimentado sólidamente su identidad; por tales condiciones, el estudio de la enseñanza de la bioética es particularmente provechoso porque se enfrenta explícita y críticamente con esta problemática.

\section{EL CURRÍCULUM OCULTO Y SU IMPORTANCIA PARA EFECTIVIZAR LA FORMACIÓN BIOÉTICA}

Muchos autores han planteado que la adquisición de diferentes tipos de valores, actitudes y comportamientos de los estudiantes son probablemente más fáciles de adquirir, a través del ejemplo o modelo que presente el profesor, en el llamado currículo oculto.

Este currículo oculto, lo define Posner (1998) como un conjunto de normas, costumbres, creencias, símbolos y valores no reconocidos o planteados explícitamente en el currículo oficial, puede tener diferentes propósitos, contenidos y repercusiones, según sea la población en la que se presente, por lo que no existen unos contenidos preestablecidos claros y una forma específica de enseñanza del mismo. Por su parte, Vallaeys (2013) refiere que el currículo oculto es un concepto central para la problemática ética en la enseñanza.
$\mathrm{Al}$ respecto, expresa que "solo una reflexión y un diagnóstico sobre la gestión institucional, el clima laboral y académico vivido cotidianamente en la universidad, permiten esperar, develar y transformar poco a poco el currículo oculto en el aula y el Ethos oculto en la universidad".

\section{COMPETENCIAS QUE PERMITEN DESARROLLAR LA ENSEÑANZA DE LA BIOÉTICA}

Las competencias bioéticas que habrían de desarrollarse en los estudiantes, deben de perdurar para toda la vida y ser aplicables en todos los contextos: familiares, sociales o en el ámbito profesional en el que se desempeñen.

\section{Competencias cognitivas y procedimentales}

En este tipo de competencia, los objetivos se orientan a la información, que en el caso de la bioética es muy amplia, constituyendo ello el cuerpo de la disciplina y el componente básico que propicia la adquisición de habilidades necesarias para analizar y fundamentar posiciones, distanciándose así de la sola opinión y permitirán al educando encarar y elucidar los dilemas morales implícitos en el quehacer cotidiano del ejercicio de su profesión.

\section{Competencias actitudinales}

A través de estas se podrán desarrollar actitudes de tolerancia, respeto, discusión racional y desarrollo de un perfil solidario, elementos que le servirán como herramientas básicas para enfrentar con solvencia las situaciones problemáticas que le plantee la ciencia, tecnología e información.

\section{METODOLOGÍAS Y ESTRATEGIAS EN LA ENSEÑANZA DE LA BIÓTICA}

La bioética se caracteriza, principalmente, por emplear un análisis racional de las situaciones conflictivas que se derivan de la aplicación tecnocientífica y las normas éticas, morales, sociales e individuales; por el empleo correcto y pleno de información concerniente al tema o caso que se trate; por su trabajo interdisciplinario, diálogo y argumento además de 
la búsqueda de consenso y responsabilidad solidaria ante líneas de investigación que puedan ser peligrosas para el hombre. Entre algunas metodologías referidas dentro del estudio de investigación, podemos mencionar a las siguientes:

Metodología de la deliberación. Según Gracia (1989), permite destacar la importancia de esta metodología por cuanto mediante ella, los estudiantes podrán asumir una actitud de apertura, estar disponibles y mostrarse abiertos al debate y a la discusión, lo cual demanda la voluntad del diálogo que implica aprender a departir, escuchar y comunicarse eficazmente, virtud que debe distinguir al profesional de las ciencias empresariales, por lo que mucho servirá para la buena relación del futuro profesional empresario en sus relaciones con los demás .

Aprendizaje participativo, vivencial y grupal: En las distintas apreciaciones de los autores, hay consenso -de acuerdo al aprendizaje centrado en el estudiante- en señalar que la enseñanza de la bioética, debe ser participativa, vivencial y preferentemente grupal, pues con ello se desarrolla moralmente a los estudiantes en sus intercambios argumentativos a partir de casos que ellos requieren aclarar y analizar.

\section{PERFIL DOCENTE PARA LA BIOÉTICA}

León correa, citado por Villa (2013), fundamenta que la enseñanza de la bioética debe cumplir con tres objetivos y a la vez retos para el docente: entregar conocimientos desde una visión interdisciplinar sobre los ámbitos complejos de que trata la bioética, modificar actitudes y comportamientos en los estudiantes e incidir en la relación que se establece entre el profesional y los otros miembros de la empresa, además de transmitir los valores éticos más apropiados y necesarios para los futuros profesionales y la sociedad en general.

En este sentido, la formación de los docentes deberá reunir mínimamente las siguientes condiciones: actitud de apertura, voluntad de diálogo, trabajo en equipo multidisciplinario, estudio sistémico, ética de responsabilidad solidaria y disposición para la constante revisión del análisis sobre temáticas diversas.

\section{MARCO INSTITUCIONAL DE LOS CENTROS UNIVERSITARIOS SANMARCOS Y CULAGOS}

La Universidad Nacional Mayor de San Marcos, de Perú, tiene su sede en el departamento de Lima y pertenece al distrito de Lima Cercado. Habiendo sido la gestora de la creación de la república del Perú, ostenta un preciado legado histórico social en sus casi cinco siglos de existencia, siendo referente importante de saberes a nivel latinoamericano. Así mismo, ha sido fuente forjadora de insignes científicos y profesionales liberales, habiendo de ella surgido los lineamientos para la creación de nuevas universidades en el país.

De acuerdo a su compendio estadístico 2014, cuenta con un total de 42,703 estudiantes, 30,979 de nivel Pregrado y 7,608 del nivel Posgrado, distribuidos entre sus veinte facultades, las cuales están agrupadas en seis áreas académicas: Ciencias Básicas, Ciencias de la Salud, Ciencias Sociales, EconómicoEmpresariales, Humanidades e Ingenierías; así mismo, cuenta con sesenta y cinco carreras profesionales.

El Área Económico Empresarial, que es objeto del análisis del presente estudio, específicamente la conforman tres Facultades que a su vez, albergan nueve carreras profesionales, siendo estas: Contabilidad, Gestión Tributaria y Auditoría Empresarial y del Sector Público, Administración, Administración de Turismo, Negocios Internacionales, Economía, Economía Pública, Economía Internacional.

Por su parte, la Universidad de Guadalajara, de México, tiene su sede en el Estado de Jalisco, ciudad de Guadalajara. Cuenta con una rica tradición histórica de más de dos siglos y es la institución de educación superior pública más importante del occidente de México y la segunda, a nivel nacional.

Esta universidad constituye una gran red universitaria que según su estadística institucional, atiende al 2014 una población total de 241,744 estudiantes, distribuidos en sus seis centros metropolitanos de formación temáticos y nueve centros universitarios regionales; entre ellos, el Centro Universitario de Los Lagos - CULAGOS, materia del presente estudio; además, de cincuenta y cinco Escuelas preparatorias, setenta y un módulos, treinta extensiones y 
un Sistema de Universidad Virtual. En cuanto a la distribución de su población estudiantil: 109,375 se encuentran en el nivel superior, el cual lo conforman un pregrado que a su vez, considera la formación en técnico superior universitario (1,370 Estudiantes) y licenciaturas (101,675 estudiantes) y un Posgrado que atiende un total de 6,330 estudiantes. Por otra parte, atienden 132,369 estudiantes en el nivel medio superior conformado por 4 Bachilleratos.

El Centro Universitario de Los Lagos-CULAGOS constituye uno de los nueve centros regionales universitarios de esta universidad que alberga a 2,526 estudiantes distribuidos en quince licenciaturas 0 carreras ofertadas, de las cuales pertenecen al área empresarial: Administración e Ingeniería en Administración Industrial.

\section{MARCO GENERAL DEL ROL ASUMIDO EN TÉRMINOS DE RESPONSABILIDAD SOCIAL UNIVERSITARIAEN SAN MARCOSY CULAGOS}

En lo que respecta al establecimiento de un marco general que refleje un rol en términos de responsabilidad social universitaria (RSU) en las carreras profesionales del área empresarial de los centros universitarios de San Marcos y CULAGOS, la indagación dentro de la investigación, permitió conocer los siguientes aspectos: Actualmente en San Marcos ya se están concretando acciones al respecto, que permitirán efectivizar el compromiso de la vinculación con la sociedad para la creación, la transmisión, la aplicación y el intercambio de conocimientos que promoverán la construcción de una sociedad solidaria y equitativa, dejando así de mantener simplemente una declaración de voluntad, sin apoyo de acciones concretas.

El aporte al desarrollo humano por parte de estas universidades se debe fundamentar en la necesidad de que la educación universitaria fortalezca su responsabilidad social y se oriente al desarrollo humano sostenible y racional; señalando su compromiso con la contribución al desarrollo educativo, así la universidad asumiría el deber de contribuir al desarrollo sostenible educativo, mejorando la formación docente, currículos e investigación sobre educación.
En este marco general, como ha sido expresado, cabe afirmar que existe una intencionalidad manifiesta por parte de ambas instituciones, a través de sus documentos de gestión, en construir una responsabilidad social dentro de su currícula es decir, pareciera que estos centros comprenden perfectamente su papel en la sociedad, por lo que plasman sus objetivos en sus documentos institucionales. Sin embargo, adolecen de programas que dinamicen y lleven a la práctica esta intencionalidad.

\section{RETOS Y PERSPECTIVAS PARA LA FORMACIÓNENBIOÉTICAEN SANMARCOS Y CULAGOS}

En este sentido, se busca brindar un aporte para que se posibilite la aplicación del enfoque bioético en el desarrollo de una perspectiva concreta de RSU, poniendo en evidencia la necesidad de trabajar desde una visión de defensa de los derechos, la defensa de una educación para la vida, el reconocimiento de los deberes ciudadanos, la protección de toda forma de vida, del medio ambiente, la formación integral del ser humano, la gestión del conocimiento con sentido social, entre otros aspectos que defiende y promueve la bioética.

El contenido bioético y de responsabilidad social universitaria también se contemplan en el currículo oculto y en los planes de estudio formales, marcando con ello, transcendencia en el cambio de la forma de pensar del egresado autónomo que, finalmente, es quien interviene en la toma de decisiones en los campos de la actividad pública y privada donde se desempeñe.

De esta manera, cada una de las materias del plan de estudios contribuiría a la formación del criterio ético y de la conducta moral de los futuros profesionales del área de los negocios, ya que se obtendrá un cambio en la concepción y aplicación de la Bioética en las nuevas generaciones.

\section{La formación de los docentes del área empresarial para la enseñanza de la bioética}

La formación del personal de la plana docente de las carreras del Área Empresarial, constituye otra de las tareas para la concreción de la enseñanza en 
bioética al interior de la universidad, por el perfil que caracteriza a sus docentes, sobre todo en el área contable.

El estilo de liderazgo que desarrollan los docentes delárea contable se orienta más a la categoría de "roles de tarea" que fundamenta la teoría de roles, próxima a la conductual, señalada en las investigaciones de la Universidad de Ohio, las cuales definen este tipo como de naturaleza cognitivos y centrados en la planificación y la organización, por lo que el planteamiento inmediato sería la innovación en sus perfiles, siendo ello condición determinante para asumir la enseñanza de la bioética.

\section{CONCLUSIONES}

1. La Universidad Nacional Mayor de San Marcos cuenta con un marco ideológico y conceptual que le da soporte a sus planteamientos sobre Responsabilidad Social Universitaria, lo cual constituye un marco favorable para la incorporación de la bioética.

2. El Centro Universitario de Los Lagos (CULAGOS) presenta en sus documentos diversa información y planteamientos que aluden explícitamente a la Responsabilidad Social Universitaria así también, hay acciones que se relacionan con algunas necesidades de la sociedad, más en la realidad concreta, estas se presentan dispersas, evidenciándose ausencia de programas formales que reflejen lo planteado.

3. El nivel de conocimiento de los estudiantes en torno a conceptos bioéticos es bajo, ello se evidencia en la mayoritaria opinión de conceder mayor importancia a un éxito empresarial en términos económicos que en términos de responsabilidad social.

\section{RECOMENDACIONES}

1. Sería altamente beneficioso para los centros universitarios: San Marcos y CULAGOS que incorporen la bioética como marco válido en la determinación y concreción de lo que cada entidad configure como responsabilidad social universitaria haciéndola explícita y aplicándola en la gestión de esta, de manera transversal y articulada en sus funciones misionales, pues ella contribuirá a definir lineamientos contundentes en la forma de gestionar sus impactos en la sociedad.

2. Se recomienda gestionar la implementación de la enseñanza de la bioética como componente de formación integral de los estudiantes del área empresarial, considerando su transversalidad en el plan curricular de las carreras del área y promover que ello se de en todos los demás programas académicos de la universidad, dada su condición de disciplina interdisciplinaria con ello, se formará no solo ciudadanos competentes en el saber y el hacer si no también en el ser, ciudadanos críticos y creativos, capaces de entender la realidad y transformarla para bien de la humanidad.

3. Se recomienda considerar la apertura de programas de sensibilización y capacitación en bioética para los docentes del área empresarial a fin de superar la debilidad curricular en este aspecto y se apoye una orientación pedagógica con claridad en este campo para tenerla en cuenta en la docencia cotidiana. 


\section{REFERENCIAS BIBLIOGRÁFICAS}

1. BETANCUR,J.H. \& NARANJO,L.A.La ética de la responsabilidad social empresarial. Disponible en: Caravedo, B. (2011). Desarrollo Sostenible, ética filantropía y responsabilidad social. En Banco Interamericano de Desarrollo. Disponible en: www.fomin.org.

2. GRACIA, D. (1989). Fundamentos de Bioética. Eudema, Madrid.

3. LÓPEZ, S. \& RIVERA-Cruz, B. (2012). El Razonamiento bioético y la orientación hacia la responsabilidad social empresarial de los estudiantes e negocios. Puerto Rico.

4. México, Universidad de Guadalajara (2014). Estadísticas Institucionales 2013-2014. Jalisco.

5. Perú, Universidad Nacional Mayor de San Marcos (2014). Modelo de Responsabilidad Social de San Marcos. Lima.

6. Perú, Universidad Nacional Mayor de San Marcos (2014). Modelo Educativo de San Marcos. Lima.

7. Perú, Universidad Nacional Mayor de San Marcos (2014). Compendio Estadístico 2014. Lima: Autor. Perú, Universidad Nacional Mayor de San Marcos - Facultad de Ciencias Administrativas (2012). Plan Estratégico Institucional 20122021. Lima.
8. Perú, Universidad Nacional Mayor de San Marcos - Facultad de Ciencias Contables (2012). Plan Estratégico Institucional 2012-2021. Lima.

9. RAMOS, N. R. (2010). Desarrollo del Enfoque en Bioética Global para la Responsabilidad Social Universitaria en la Fundación Universidad Autónoma de Colombia. Tesis de Magíster para optar el grado de Magíster en Administración de Empresas. Facultad de Ciencias Económicas, Universidad Nacional de Colombia. Colombia.

10. VALLAEYS, F. (2014). La responsabilidad social universitaria: un nuevo modelo universitario contra la mercantilización. Disponible en: http:// ries.universia.net

11. VALLAEYS, F. (2013). Breve marco teórico de Responsabilidad Social Universitaria. Disponible en http://www.jadb.org/etica.

12. VILLA, J. A. (2013). Fundamentos para estructurar e implementar un programa académico universitario en bioética odontológica en Colombia. Disponible en:

13. VILLEGAS, E. M. (2013). Significados sobre bioética que emergen entre actores del currículo de medicina de la Universidad de Antioquía. Colombia. Disponible en: http://www.bioeticaunbosque.edu.co/Investigacion/tesis/ELSA_MARIA_VILLEGAS.pdf 